\title{
AD Robotics - Long Reach Robotic Arm
}

\section{Amanda Hoeksema \& Brenda Sanchez \\ Under the mentorship of Kris Anderson}

\section{The Issue With Maintenance}

Vital systems for the accelerator's operation are tucked up and behind components, obstructing them from easy view; this makes it challenging to identify the accurate locations of broken parts. A balanced, long reach robotic arm composed of multiple links capable of maneuvering around obstructions is the solution to this problem.

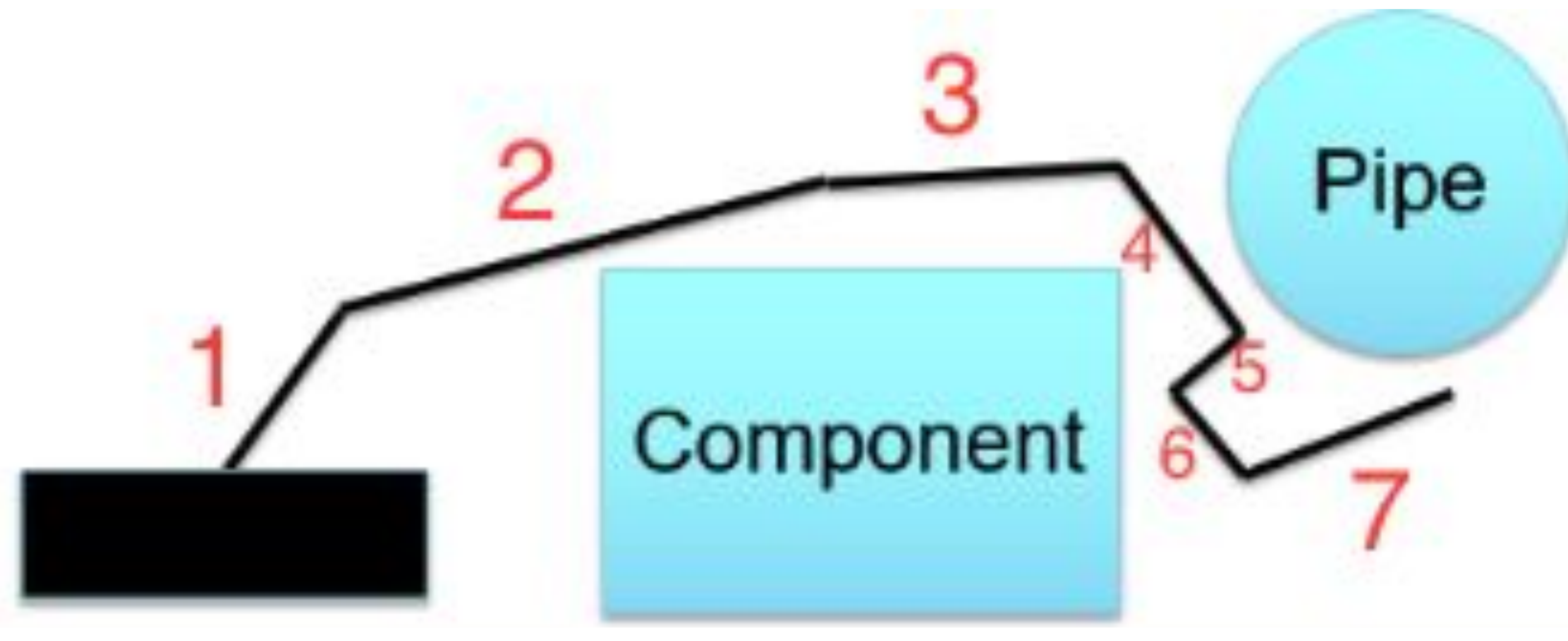

Sample diagram of how the arm would navigate around obstacles.

\section{The Solution}

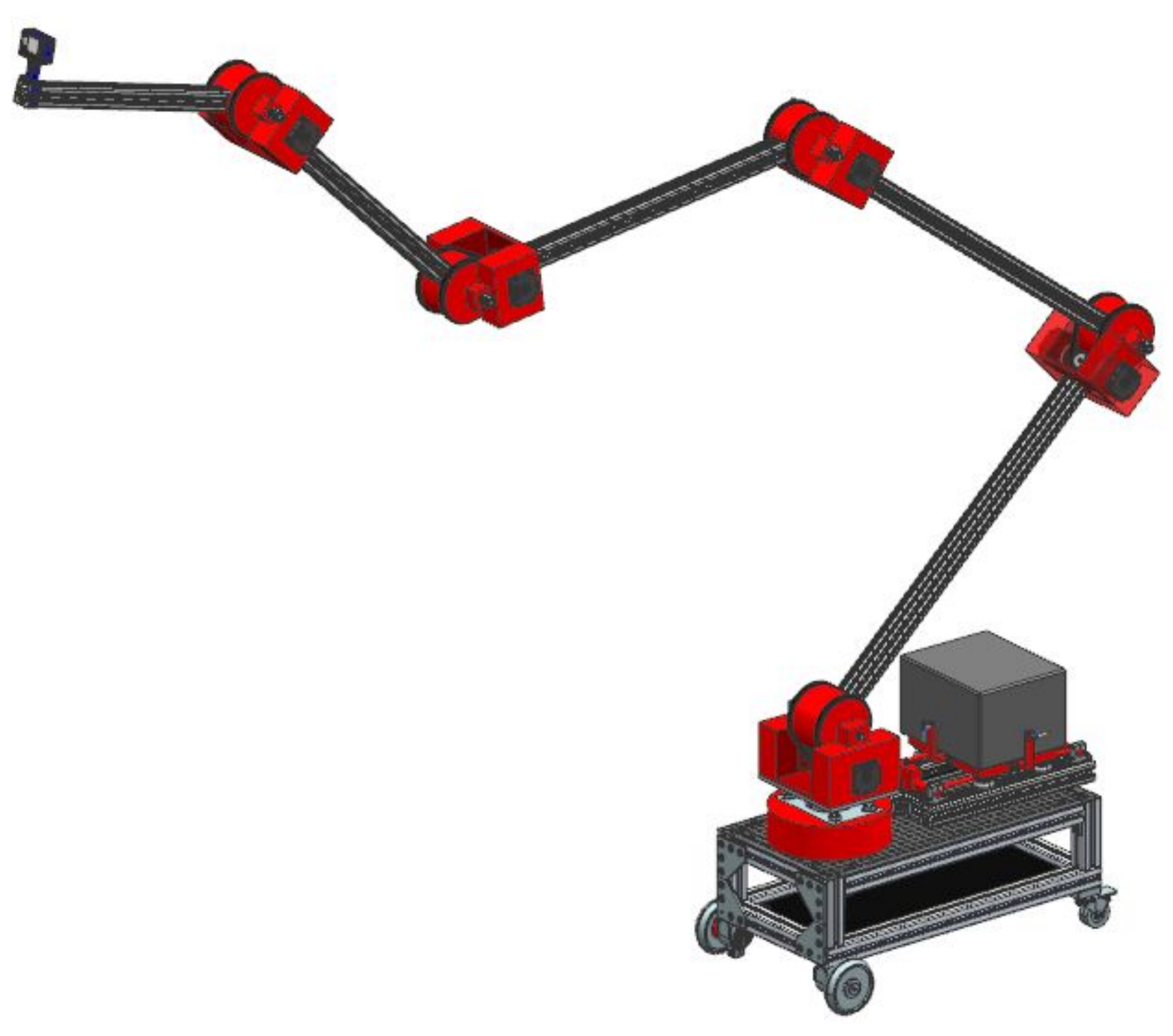

Full robot arm assembly mounted to a symbolic base.

The resulting robot design is comprised of five 80/20 links each with $180^{\circ}$ of rotational freedom balanced with a 110 Ibs steel counterweight. The counterweight and arm assembly are an independent system, meaning they can be removed from the depicted wheeled base and transferred to a different base without difficulty. Each link rotates separately from the other, allowing for more controlled movement around accelerator components all-the-while maintaining balance through the counterweight.

\section{How the Arm is Balanced}

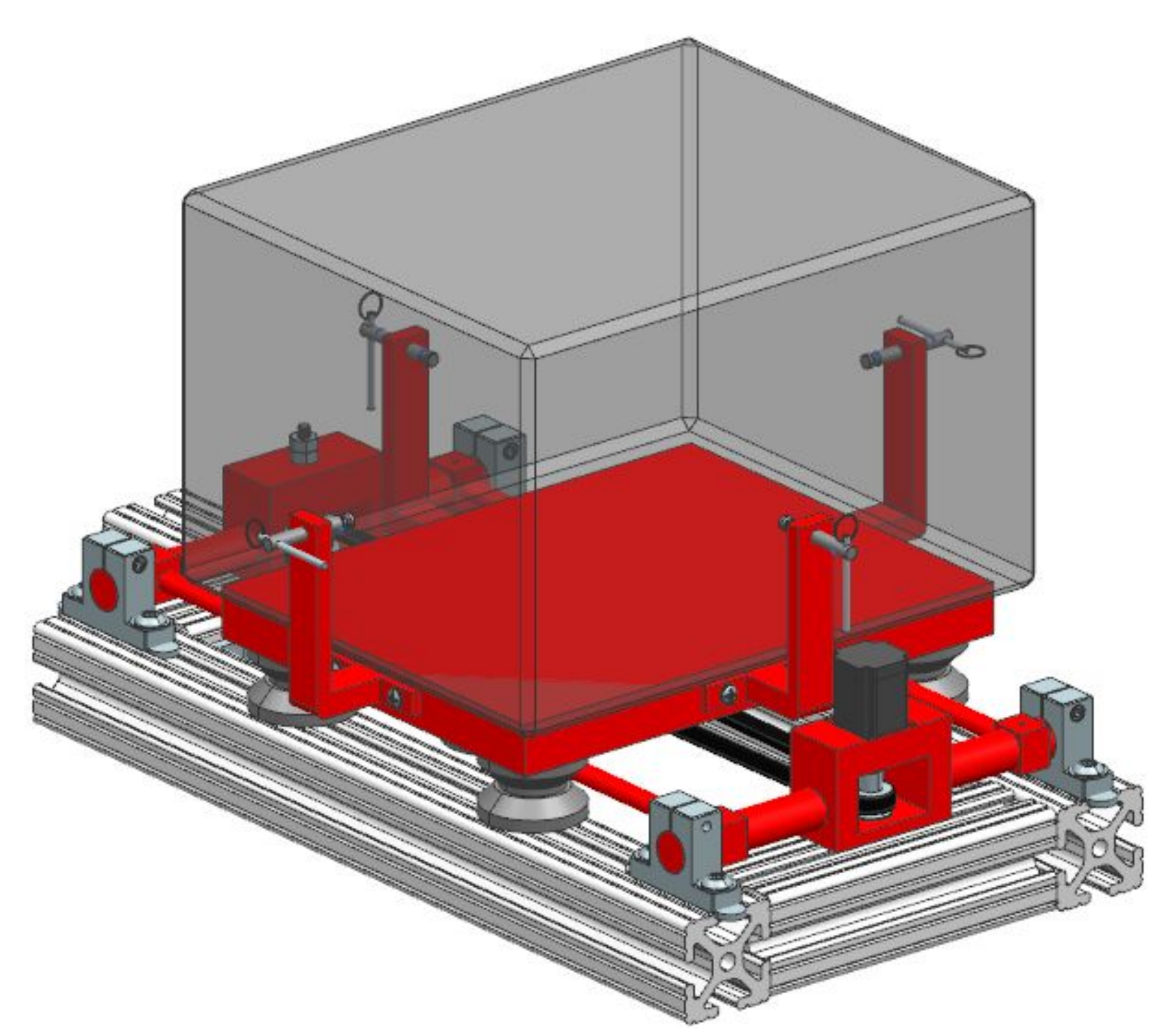

The counterweight design centers around mobility; it needed to have freedom of one-dimensional motion to counteract the outward extension of the arm links. Supported by an 80/20 frame and powered by a NEMA 23 stepper motor rotating a belt and pulley system, the counterweight translates across a span of 11 inches. The weight is fastened in place with four threaded screws with padded ends to prevent the steel from slipping during movement. The complete model affixes to a slot in the lowest component of the arm link assembly.

Full image of sliding system with the counterweight made transparent for ease of view.
Two NEMA 23 stepper motors individually power each 80/20 1515-48 arm link. Each joint rotation depends on a pulley system: a timing belt wraps around a motor pulley and the 3D printed arm joint. The joints are supported by a 7 inch threaded bolt with two jam nuts at the end, preventing rotation but allowing for linear adjustment. A 1.5 " $\times 1.5^{\prime \prime}$ cut extrusion is made on the joints' face to fasten the $80 / 20$ beams. The joint ases are 3D printed to create an open extrusion to store the motors and an opening for the motor shaft. On the top extrusion are threaded holes joint. Lastly, a 3D printed GoPro camera mount is fastened onto the end of the final arm link.
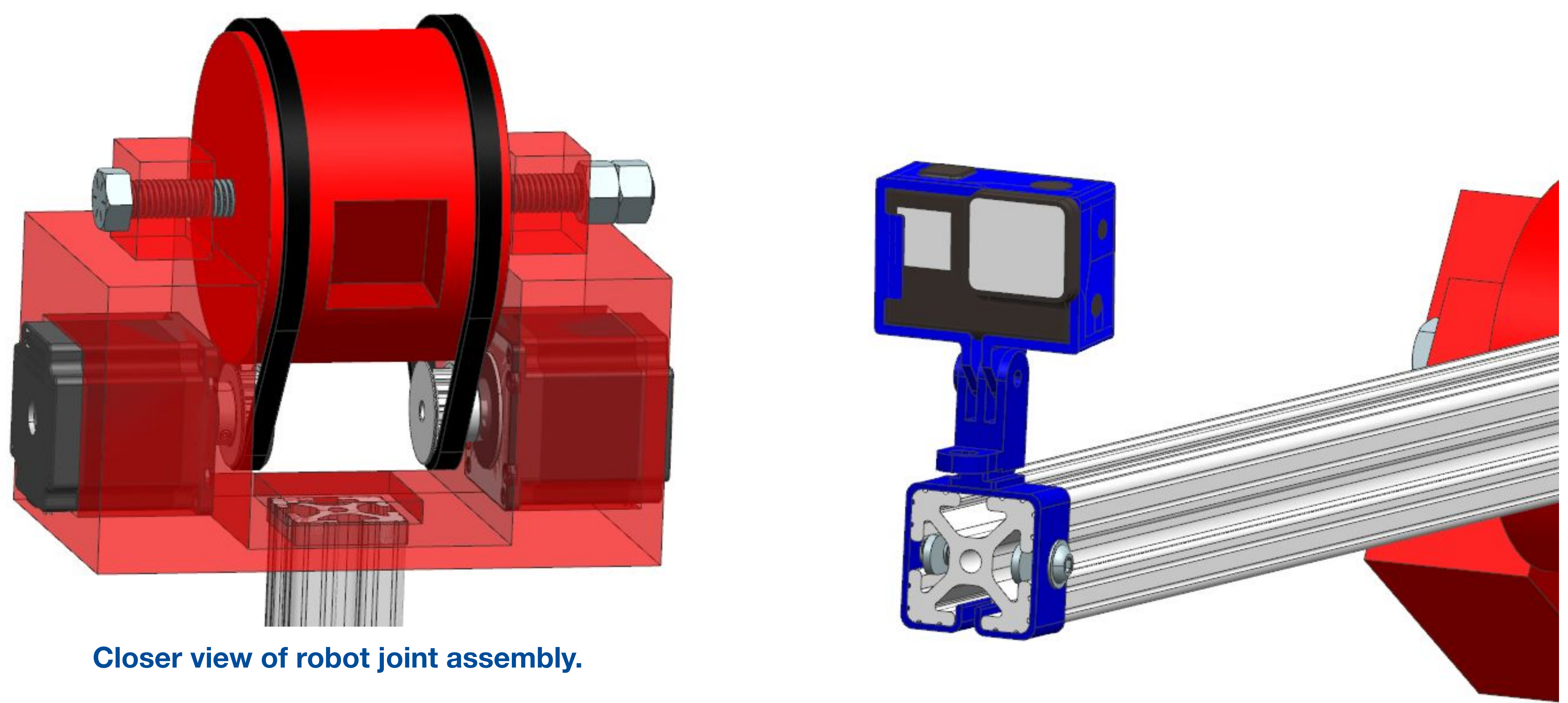

GoPro camera assembly mounted onto 80/20.

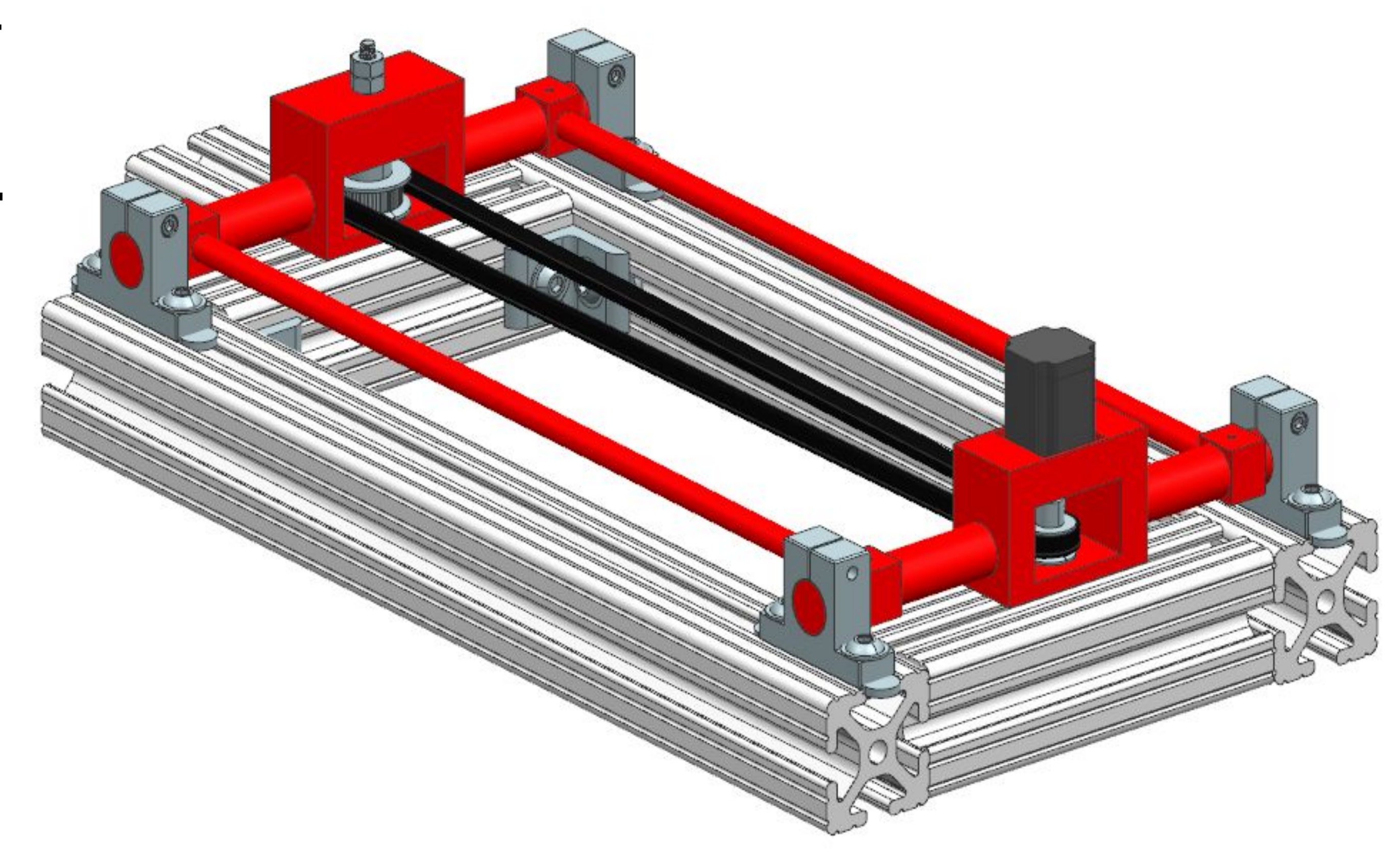

View of stepper motor, belt, pulley system. 